\title{
Genetic and hormonal control of hepatic steatosis in female and male mice ${ }^{1 \text { s }}$
}

\author{
Frode Norheim, ${ }^{2}{ }^{*}$ Simon T. Hui,* Emre Kulahcioglu,* Margarete Mehrabian, ${ }^{*}$ Rita M. Cantor, ${ }^{\dagger}$ \\ Calvin Pan, ${ }^{*}$ Brian W. Parks, ${ }^{\S}$ and Aldons J. Lusis ${ }^{2} *$ \\ Department of Medicine, * Division of Cardiology, University of California at Los Angeles, Los Angeles, CA; \\ Department of Human Genetics, ${ }^{\dagger}$ David Geffen School of Medicine at University of California at Los Angeles, \\ Los Angeles, CA; and Department of Nutritional Sciences, ${ }^{\S}$ University of Wisconsin-Madison, Madison, WI
}

Abstract The etiology of nonalcoholic fatty liver disease is complex and influenced by factors such as obesity, insulin resistance, hyperlipidemia, and sex. We now report a study on sex difference in hepatic steatosis in the context of genetic variation using a population of inbred strains of mice. While male mice generally exhibited higher concentration of hepatic TG levels on a high-fat high-sucrose diet, sex differences showed extensive interaction with genetic variation. Differences in percentage body fat were the best predictor of hepatic steatosis among the strains and explained about $30 \%$ of the variation in both sexes. The difference in percent gonadal fat and HDL explained $9.6 \%$ and $6.7 \%$ of the difference in hepatic TGs between the sexes, respectively. Genome-wide association mapping of hepatic TG revealed some striking differences in genetic control of hepatic steatosis between females and males. Gonadectomy increased the hepatic TG to body fat percentage ratio among male, but not female, mice. Our data suggest that the difference between the sexes in hepatic TG can be partly explained by differences in body fat distribution, plasma HDL, and genetic regulation.j. Future studies are required to understand the molecular interactions between sex, genetics, and the environment.-Norheim, F., S. T. Hui, E. Kulahcioglu, M. Mehrabian, R. M. Cantor, C. Pan, B. W. Parks, and A. J. Lusis. Genetic and hormonal control of hepatic steatosis in female and male mice. J. Lipid Res. 2017. 58: 178-187.

Supplementary key words genetics $\bullet$ nonalcoholic fatty liver disease $\bullet$ obesity $\bullet$ insulin $\bullet$ hormones $\bullet$ nutrition/lipids $\bullet$ gonadectomy

Nonalcoholic fatty liver disease (NAFLD) is the most common liver disease in Western developed countries (1) and nonalcoholic steatohepatitis (NASH) is projected to become the leading cause of liver transplantation in the USA

This work was supported by Foundation for the National Institute of Health Grants HL28481, HL114437, and HL30568, and Transatlantic Network of Excellence Award 12CVD02. Additional support was provided by Research Council of Norway (240405/F20) (to F.N.). The content is solely the responsibility of the authors and does not necessarily represent the official views of the $\mathrm{Na}$ tional Institutes of Health. The authors declare that they have no competing financial interests.

Manuscript received 16 August 2016 and in revised form 17 October 2016.

Published, JLR Papers in Press, November 3, 2016

DOI 10.1194/jlr.MO71522 by 2020 (2). By some estimates, $50 \%$ of Americans will have NAFLD by 2030 (3). Population studies have shown that NAFLD is associated with obesity, visceral adiposity, insulin resistance, dyslipidemia, sex, age, and ethnicity $(4,5)$. Because most people with NAFLD suffer from obesity and insulin resistance, treatments aim at weight reduction, control of dyslipidemia, and improving insulin sensitivity through lifestyle changes and pharmacological agents, such as metformin, statins, fibrates, and thiazolidinediones (6).

NAFLD encompasses many abnormalities, ranging from accumulation of fat within hepatocytes (steatosis), to NASH, fibrosis, and cirrhosis in the absence of excessive consumption of alcohol and hepatic viral infection. Advanced NAFLD can eventually progress to end-stage liver disease with increased risk of hepatocellular carcinoma (7). Factors determining the progressive phenotype of NAFLD remain elusive. However, environmental factors (such as diet and lifestyle), genetic variations, ethnicity, and sex can play important roles in determining the disease phenotype and progression (8-12). Whereas the most common cause of NAFLD probably can be attributed to energy intake exceeding

Abbreviations: Atoxl, antioxidant $1 ; \mathrm{BF} \%$, body fat percentage; DAVID, Database for Annotation, Visualization, and Integrated Discovery; eQTL, expression quantitative trait locus; $G c k r$, glukokinase regulator; Gde1, glycerophosphodiester phosphodiesterase 1; GO, gene ontology; $G p x 4$, glutathione peroxidase 4; GWAS, genome-wide association study; HF/HS, high-fat high-sucrose; HMDP, Hybrid Mouse Diversity Panel; Hnrnpab, heterogenous nuclear ribonucleoprotein A/B; HOMA-IR, homeostatic model assessment of insulin resistance; Invs, inversin; LD, linkage disequilibrium; Lyplal1, lysophospholipase-like 1; Mboat7, membrane bound $O$-acyltransferase domain containing 7; Ncan, neurocan; NAFLD, nonalcoholic fatty liver disease; NASH, nonalcoholic steatohepatitis; Phykpl, 5-phosphohydroxy-L-lysine phosphor-lyase; Skp1a, s-phase kinase protein 1a; $\operatorname{Tm} 6 s f 2$, transmembrane 6 superfamily member 2; Trib1, tribbles pseudokinase 1; Zfp354a, zinc finger protein 354a.

'The data discussed in this publication have been deposited in NCBI's Gene Expression Omnibus (Norheim et al., 2016) and are accessible through GEO Series accession number GSE64770 (http://www.ncbi. nlm.nih.gov/geo/query/acc.cgi?acc=GSE64770).

${ }^{2}$ To whom correspondence should be addressed.

e-mail: FNorheim@mednet.ucla.edu (F.N.); JLusis@mednet.ucla. edu (A.J.L.).

s The online version of this article (available at http://www.jlr.org) contains a supplement.

Copyright ( $\odot 2017$ by the American Society for Biochemistry and Molecular Biology, Inc. 
rates of energy expenditure, the heritability of hepatic steatosis is estimated to be $\sim 52 \%$ in a human twin study (13). However, only a small fraction of genes accounting for hepatic steatosis and advanced liver disease have been identified. The frequency of fatty liver is also associated with ethnicity (4). Although the higher prevalence of NAFLD in Hispanics can be explained by higher prevalence of obesity and insulin resistance, the lower rate of NAFLD in blacks cannot be explained by common risk factors (4). While there appear to be clear differences in hepatic steatosis between sexes, the underlying mechanisms are unclear. Hepatic steatosis is reported to be more prevalent in white men than in white women $(4,12)$. NASH has been reported to be more prevalent in females than in males (11), and lean women appear to have a higher prevalence of NAFLD than lean men (14). Also, NAFLD has been shown to be more prevalent in post- as compared with premenopausal women, suggesting that sex hormones may influence the onset of NAFLD (15). The fact that breast cancer patients treated with an estrogen receptor antagonist develop massive hepatic steatosis and even typical NASH indicates that estrogen affects development of this disease (16). Cross-sectional studies assessing the association between plasma testosterone and NAFLD have shown conflicting results (17-19). However, rodent studies have shown that both estrogen and testosterone can have an inhibitory effect on liver lipid accumulation $(20,21)$.

The aim of this study was to describe the sex difference in hepatic steatosis in a population of diverse inbred strains of mice known as the Hybrid Mouse Diversity Panel (HMDP) fed a high-fat high-sucrose (HF/HS) diet (22). The use of the HMDP provided a rich genetic background to assess both the phenotypes and genetic interactions associated with hepatic TG concentration in mice of both sexes.

\section{MATERIALS AND METHODS}

\section{Animal procedures}

All 100 female and 113 male mouse strains included in the study were obtained from Jackson Laboratory and have been described in detail previously $(23,24)$. The experimental design of the HF/ HS feeding study has also been described previously (25). Briefly, the female and male strains included in the HMDP study were maintained on a chow diet (Ralston Purina Co.) until 8 weeks of age before switching over to a HF/HS diet (Research Diet-D12266B; New Brunswick, NJ) for 8 weeks. The macronutrient composition of the HF/HS diet was as follows: $16.8 \%$ kcal protein, $51.4 \% \mathrm{kcal}$ carbohydrates, and $31.8 \% \mathrm{kcal}$ fat. The mice were euthanized after a $4 \mathrm{~h}$ fast at approximately the same time of the day (between 10:30 $\mathrm{AM}$ and noon). Plasma, liver, and subcutaneous, mesenteric, gonadal, and retroperitoneal adipose tissue were flash-frozen in liquid nitrogen immediately after harvesting and stored at $-80^{\circ} \mathrm{C}$. The mice were housed in a $14 \mathrm{~h}$ day and $10 \mathrm{~h}$ night cycle. The animal procedures were approved by the Institutional Care and Use Committee of the University of California, Los Angeles.

\section{Gonadectomy and ovariectomy}

The gonadectomy and ovariectomy study was described in detail previously (25). Briefly, the female and male mouse strains (C3H/ HeJ, C57BL/6J, and DBA/2J) were either maintained on chow diet for 16 weeks or maintained on a chow diet until 8 weeks of age before switching over to a HF/HS diet for 8 weeks. The mice were gonadectomized under isoflurane anesthesia at 6 weeks of age. Control mice were either sham operated under isoflurane anesthesia or left untreated. There were three to four mice per group.

\section{Body composition and adipose depots}

Total body fat mass and lean mass were measured by magnetic resonance imaging using Bruker Minispec, as described (26). The subcutaneous, mesenteric, gonadal, and retroperitoneal adipose tissues were carefully dissected from each mouse and weighed before being flash-frozen in liquid nitrogen.

\section{Hepatic lipid isolation}

Lipids from 388 and 478 livers of female and male mice, respectively, were extracted by methods described by Folch, Lees, and Sloane-Stanley (27). Around $100 \mathrm{mg}$ of liver (wet weight) was used for the Folch extraction. The dried organic extract was dissolved in $1.8 \%$ (wt/vol) Triton X-100. A colorimetric assay from Sigma (St. Louis, MO) and Wako (Richmond, VA) were used to quantify TG and phospholipids, respectively. Total cholesterol and unesterified cholesterol were measured with in-house reagents, as described previously (28). Two to eight livers were included per female strain depending on accessibility and variation in the data. The number of livers per female strain is included in supplemental Table 1 . The number of livers done per male strain has been described previously (24).

\section{Plasma insulin, glucose, and lipids}

Blood was collected from mice after a $4 \mathrm{~h}$ fast using retroorbital bleeding under isoflurane anesthesia. The plasma levels of insulin (Alpco Diagnostics, Salem, NH), glucose (Stanbio Laboratory, Boerne, TX), TG (Sigma), total cholesterol, and HDL were measured as described previously (28). A colorimetric assay was used to quantify plasma glycerol (Sigma). Homeostatic model assessment of insulin resistance (HOMA-IR) was calculated using the equation: (glucose $\times$ insulin) $/ 405$.

\section{RNA isolation and global gene expression analysis}

RNA was isolated from liver and gonadal adipose tissue as described previously (24). Briefly, tissue was homogenized in Qiazol and RNA was isolated using RNeasy columns (Qiagen, Valencia, CA). Global gene expression was analyzed using Affymetrix HT_MG430A arrays, and data were filtered as described previously (23). Gene ontology (GO) analyses were performed using the Database for Annotation, Visualization, and Integrated Discovery (DAVID) database (23). Only GO groups that were statistically significant after Bonferroni correction were included.

\section{Association analysis}

Genotypes for all 100 female and 113 male strains were obtained from the Jackson Laboratory using the Mouse Diversity Array (29). SNPs that had poor quality or had a minor allele frequency of more than $5 \%$ and a missing genotype rate of less than $10 \%$ were removed. After filtering, 200,000 SNPs were left. Genome-wide association of hepatic TG and adipose and liver mRNA expression was performed using Factored Spectrally Transformed Linear Mixed Models, which use a linear mixed model to correct for population structure (30). A cut-off value for genome-wide significance was set at $3.46 \times 10^{-6}$, as determined previously for the HMDP (23).

\section{Accession numbers}

The NCBI GEO accession number for the microarray data reported in this paper is GSE64770. 


\section{Statistics}

Forward stepwise linear regression was used to identify the models that best predict TGs in male and female mice analyzed separately. We began the stepwise regression by considering all 15 potential predictors and used a 0.05 level of significance to include those that would be considered to improve prediction. The predicted TGs were log transformed. The TG predictive models were different in males and females. To better identify the traits that best predict the sex difference in TGs, the average difference between the males and females was taken for each mouse strain and for each of the 15 possible predictors, as well as the measure of TGs. These differences were used to build a model for TG sex differences using forward stepwise linear regression, as described above. Correlations were calculated with biweight midcorrelations. GO pathways calculated in DAVID were Bonferroni corrected. Unless otherwise noted values presented are expressed as mean \pm SD. Statistical differences between groups were tested using unpaired two-tailed Student's $t$-tests. Statistical analyses were performed using R statistical programming and Excel.

\section{RESULTS}

\section{Large variation in liver steatosis among inbred strains of female mice fed a HF/HS diet}

We have previously described genetic and environmental factors contributing to hepatic steatosis in 113 strains of male mice (24). To study the factors contributing to hepatic steatosis in females, we fed female mice from 100 strains of the HMDP a HF/HS diet for 8 weeks before quantifying hepatic lipid content. Lipids from 388 livers were extracted as described by Folch, Lees, and SloaneStanley (27). The accumulation of hepatic TG varied widely, with up to a 12.7-fold difference between inbred strains (Fig. 1A). Hepatic TG was strongly correlated with the concentration of total cholesterol (Fig. 1B) in the livers, and to a lesser degree to unesterified cholesterol (Fig. 1C) and phospholipids (Fig. 1D).

\section{Male mice generally accumulate more hepatic TG than female mice}

To compare the hepatic TG content between the sexes, we focused on the 98 strains of mice for which we had liver lipid data in both males and females (Fig. 2). The female mice showed less variation between the strains (up to a 12.7-fold difference) in hepatic TG than the male mice, which showed up to a 24.9-fold difference. Hepatic TG was strongly correlated between the sexes $(r=0.41, P=2.98 \times$ $10^{-5}$; supplemental Fig. S1). Most, but not all, of the male mice accumulated more TG in their livers than their female counterparts (Fig. 2). This was also true after normalization to body fat percentage (BF\%) (supplemental Fig. S2). However, when we normalized the hepatic TG content to $\mathrm{BF} \%$ the difference between the sexes was largest in the strains with a low hepatic TG to BF\% ratio (supplemental Fig. S2).

\section{Sex-dependent differences in associations between hepatic steatosis and obesity, insulin resistance, and dyslipidemia}

Obesity, adipose distribution, insulin resistance, and dyslipidemia are all potential risk factors for NAFLD (31).
To study the association of hepatic TG with relevant phenotypes collected in our HMDP, we used the 85 strains of mice that had a complete set of matching phenotypes in both males and females. Both females $(r=0.47, P=$ $\left.5.69 \times 10^{-6}\right)$ and males $\left(r=0.50, P=1.36 \times 10^{-6}\right)$ showed a strong correlation between hepatic TG and BF\% (Table 1). In females, both the subcutaneous $(r=0.37, P=4.30 \times$ $\left.10^{-4}\right)$, mesenteric $\left(r=0.29, P=6.81 \times 10^{-3}\right)$, gonadal $(r=$ $\left.0.34, P=1.28 \times 10^{-3}\right)$, and retroperitoneal $(r=0.35, P=$ $1.13 \times 10^{-3}$ ) adipose depots were correlated with hepatic TG. In the males, the associations between hepatic TG and the adipose tissues were more depot dependent (Table 1). Whereas subcutaneous $\left(r=0.43, P=4.42 \times 10^{-5}\right)$ and mesenteric $\left(r=0.33, P=1.77 \times 10^{-3}\right)$ adipose tissue showed a good correlation with hepatic TG, the gonadal and retroperitoneal adipose depots were not significantly correlated with hepatic steatosis (Table 1 ). We have previously shown that male mice are more prone to insulin resistance than females (25). When correlating hepatic TG levels with plasma insulin and HOMA-IR, female mice showed a less significant correlation with insulin $(r=0.22, P=0.045)$ and HOMA-IR $\left(r=0.30, P=5.06 \times 10^{-3}\right)$ as compared with male mice (insulin, $r=0.36, P=6.61 \times 10^{-4}$; HOMA-IR, $r=$ $\left.0.41, P=8.34 \times 10^{-5}\right)$. Glucose, on the other hand, showed a similar correlation to hepatic steatosis between the sexes (Table 1). The female mice showed more significant correlations as compared with males with respect to associations between hepatic TG and plasma lipids (Table 1).

To investigate to determine which of the phenotypes in Table 1 had the largest impact on fatty livers, we did linear regression analysis separately in both sexes. The best model in females included a $\mathrm{BF} \%$ that explained $30.2 \%$ $\left(P=7.1 \times 10^{-9}\right)$ of the variation in hepatic TG, and total plasma cholesterol additionally explained $10.3 \%(P=3.1 \times$ $\left.10^{-4}\right)$. In male mice, $\mathrm{BF} \%$ explained $31.9 \%(P=1.20 \times$ $10^{-9}$ ) of the variation in liver TG. The percentage retroperitoneal weight and glucose further explained $10.3 \%$ $\left(P=1.99 \times 10^{-4}\right)$ and $3.2 \%(P=0.033)$ of the variation in hepatic TG, respectively. Lastly, we identified a model that best explains the difference in hepatic TGs between males and females. The difference in percent gonadal fat explains $9.6 \%(P=0.003)$ of the difference in TGs and the difference in HDL explains an additional $6.7 \%$ $(P=0.012)$.

\section{Liver and adipose genes associated with hepatic TG in both sexes}

We have previously shown a significant enrichment in mitochondria genes among the transcripts with the highest correlation with hepatic TG in male mice (24). As seen for males when we performed enrichment analysis using the DAVID GO program (32) on the 1,000 liver genes mostly correlated to hepatic TG in females, we identified a significant enrichment in mitochondria genes (Fig. 3). Among the 130 genes enriched for mitochondria, 111 showed a positive association with hepatic TG. This suggests that mitochondrial function is also linked to accumulation of lipids in the liver of female mice. In addition, genes coding for organelle membranes were enriched in steatotic livers 

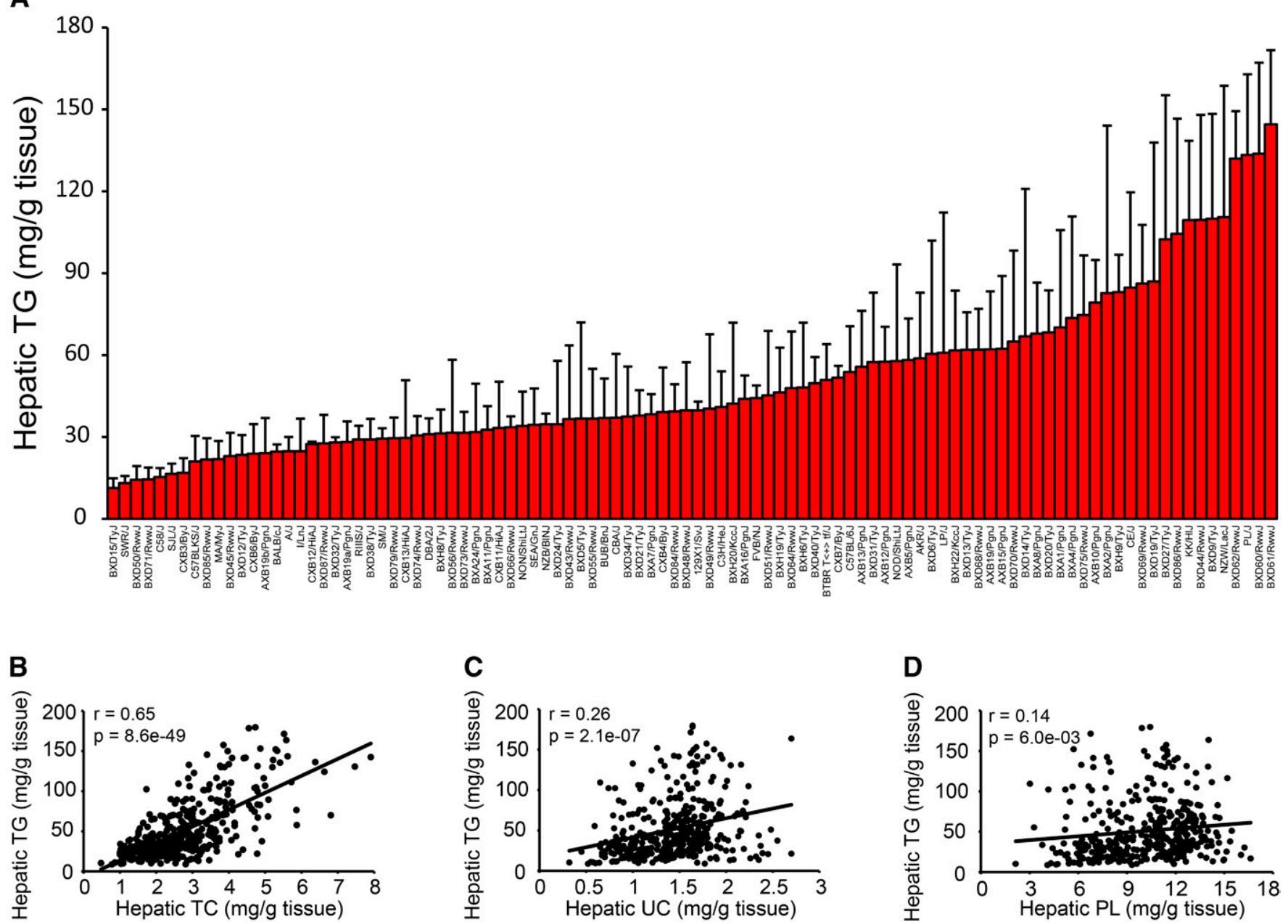

Fig. 1. Genetic variation in hepatic TG accumulation among female inbred mouse strains. A: Hepatic TG in 100 female mouse strains after 8 weeks of HF/HS feeding. Results are presented as mean \pm SD. B-D: Correlation of hepatic TG with hepatic total cholesterol (TC) (B), hepatic unesterified cholesterol (UC) (C), and hepatic phospholipids (PL) (D). r, biweight midcorrelation; $p, P$ value.

of both sexes, and genes coding for the respiratory chain were enriched in females (Fig. 3). Among the 1,000 genes most correlated to hepatic TG in females, 642 genes were also significantly correlated $(P>0.05)$ in males. Among the 25 gene transcripts that showed the strongest correlation with hepatic TG in females, 24 were significantly correlated in males (Table 2). These results suggest that the pathways underlying hepatic steatosis are largely conserved between male and female mice. Several of the genes that showed the strongest correlation with hepatic TG are involved in lipid metabolism. For example, Cd36 is well-known to bind fatty acids at the cell membrane (33) and mice heterozygous

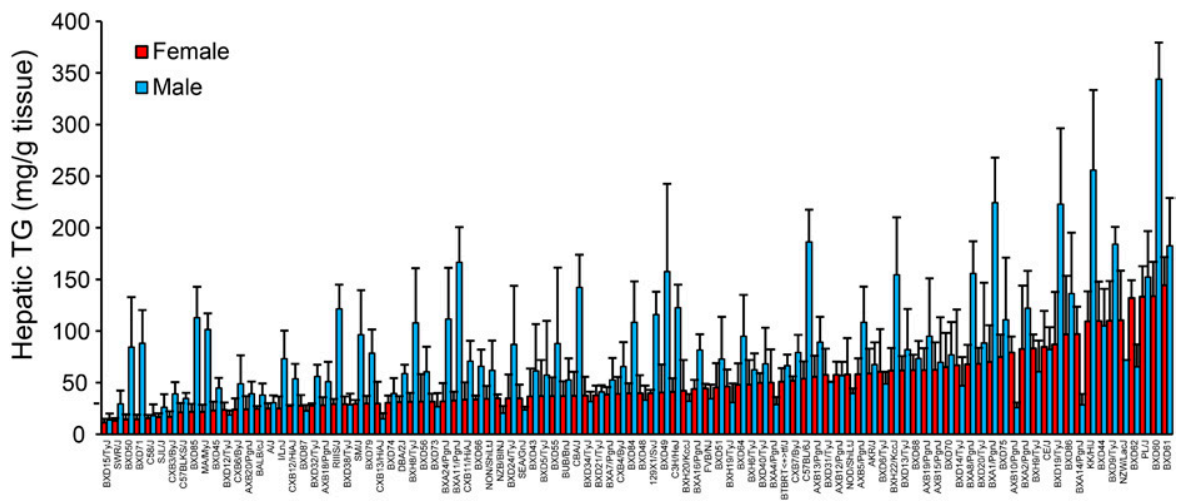

Fig. 2. Large variation in hepatic TG accumulation between the sexes. Hepatic TG in female (red) and male (blue) mice after 8 weeks of HF/HS feeding. Results are presented as mean \pm SD. 
TABLE 1. Correlation between hepatic TG and different phenotypic factors in both sexes

\begin{tabular}{|c|c|c|c|c|c|}
\hline \multirow[b]{2}{*}{ Variable } & & \multicolumn{2}{|c|}{ Female } & \multicolumn{2}{|c|}{ Male } \\
\hline & & $r$ & $P$ & $r$ & $P$ \\
\hline Body fat (\%) & $\mathrm{a}$ & 0.47 & $5.69 \mathrm{E}-6$ & 0.50 & $1.36 \mathrm{E}-6$ \\
\hline Subcutaneous fat (\%) & $\mathrm{b}$ & 0.37 & $4.30 \mathrm{E}-4$ & 0.43 & $4.42 \mathrm{E}-5$ \\
\hline Mesenteric fat (\%) & $\mathrm{b}$ & 0.29 & $6.81 \mathrm{E}-3$ & 0.33 & $1.77 \mathrm{E}-3$ \\
\hline Gonadal fat (\%) & $\mathrm{b}$ & 0.34 & $1.28 \mathrm{E}-3$ & 0.17 & 0.124 \\
\hline Retroperitoneal fat (\%) & b & 0.35 & $1.13 \mathrm{E}-3$ & -0.08 & 0.439 \\
\hline Plasma glucose (mg/dl) & & 0.29 & $6.88 \mathrm{E}-3$ & 0.29 & $8.13 \mathrm{E}-3$ \\
\hline Plasma insulin (pg/dl) & & 0.22 & 0.045 & 0.36 & $6.61 \mathrm{E}-4$ \\
\hline HOMA-IR & & 0.30 & $5.06 \mathrm{E}-3$ & 0.41 & $8.34 \mathrm{E}-5$ \\
\hline Plasma TG (mg/dl) & & -0.20 & 0.071 & -0.13 & 0.245 \\
\hline Plasma cholesterol (mg/dl) & & 0.50 & $1.34 \mathrm{E}-6$ & 0.33 & $2.24 \mathrm{E}-3$ \\
\hline HDL & & 0.46 & $1.08 \mathrm{E}-5$ & 0.31 & 4.01E-3 \\
\hline LDL & & 0.38 & $2.92 \mathrm{E}-4$ & 0.29 & $7.04 \mathrm{E}-3$ \\
\hline Plasma glycerol (mg/dl) & & 0.17 & 0.117 & 0.19 & 0.088 \\
\hline
\end{tabular}

Correlations were calculated with biweight midcorrelations. $\mathrm{a}=$ NMR data [fat $/($ fat + lean + water $) \times 100] . b=$ gram dissected fat $/$ gram total body weight.

for glutathione peroxidase 4 (Gpx4) have increased liver steatosis (34).

The mRNA expression in liver and adipose tissue of several genes identified in human genome-wide association studies (GWASs) for NAFLD showed significant correlation with hepatic TG in our mice (35-40) (Table 3). In female liver, these included membrane bound $O$-acyltransferase domain containing 7 (Mboat 7) and lysophospholipase-like 1 (Lyplal1), and in female adipose tissue these included Mboat 7 and tribbles pseudokinase 1 (Trib1). In male liver, glucokinase regulator (Gckr) and Lyplal1 showed a significant correlation with hepatic TG, and in male adipose tissue transmembrane 6 superfamily member 2 (Tm6sf2), neurocan (Ncan), and Trib1 were correlated with liver TG. In general, the correlations of the human GWAS genes with hepatic TG in mice were associated in the same direction between the sexes.

\section{Genome-wide association mapping identifies a} chromosome 11 locus for hepatic steatosis in female mice

To investigate the difference in genetic regulation of hepatic steatosis between the sexes, we performed a GWAS analysis on the hepatic TG content with approximately

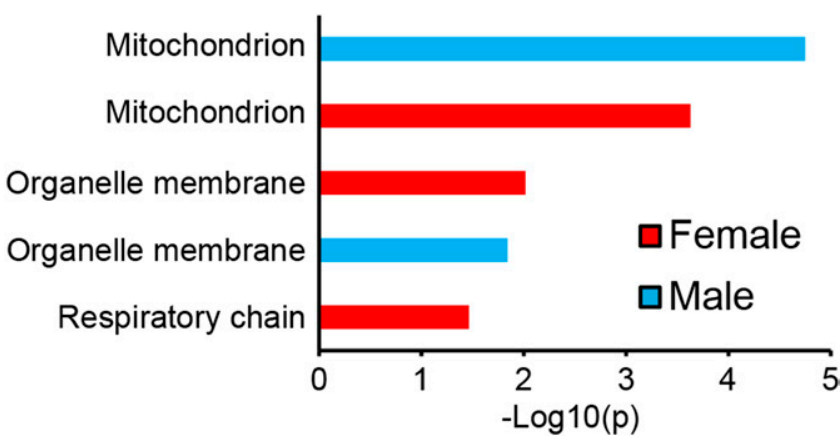

Fig. 3. Pathway enrichment analysis using the top 1,000 most correlated genes with hepatic TG. GO pathways reaching significance for female (red) and male (blue) after Bonferroni correction are shown $(P<0.05)$. GO analyses were performed using the DAVID database. The $P$ value is shown as $-\log 10$ of $\mathrm{p}$.
TABLE 2. The top 25 ranked female liver genes correlated to hepatic TG in both sexes

\begin{tabular}{|c|c|c|c|c|}
\hline \multirow[b]{2}{*}{ Gene } & \multicolumn{2}{|c|}{ Females } & \multicolumn{2}{|c|}{ Males } \\
\hline & $r$ & $P$ & $r$ & $P$ \\
\hline Ermp1 & 0.550 & $3.68 \mathrm{E}-9$ & 0.545 & $5.44 \mathrm{E}-10$ \\
\hline Entpd5 & 0.548 & $4.21 \mathrm{E}-9$ & 0.536 & $1.138 \mathrm{E}-9$ \\
\hline Cd36 & 0.540 & $8.24 \mathrm{E}-9$ & 0.695 & $1.85 \mathrm{E}-17$ \\
\hline$A b c a 8 a$ & -0.524 & $2.68 \mathrm{E}-8$ & -0.435 & $1.63 \mathrm{E}-6$ \\
\hline Gpx4 & 0.522 & $3.01 \mathrm{E}-8$ & 0.477 & $1.05 \mathrm{E}-7$ \\
\hline Anxas & 0.519 & $3.84 \mathrm{E}-8$ & 0.484 & $6.46 \mathrm{E}-8$ \\
\hline Ly6d & 0.512 & $5.98 \mathrm{E}-8$ & 0.402 & $1.14 \mathrm{E}-5$ \\
\hline Pex11a & 0.505 & $9.63 \mathrm{E}-8$ & 0.472 & $1.49 \mathrm{E}-7$ \\
\hline Emg1 & -0.497 & $1.63 \mathrm{E}-7$ & -0.495 & $2.85 \mathrm{E}-8$ \\
\hline Dek & -0.497 & $1.67 \mathrm{E}-7$ & -0.188 & 0.047 \\
\hline 9130401M01Rik & 0.494 & $2.07 \mathrm{E}-7$ & 0.501 & $1.89 \mathrm{E}-8$ \\
\hline Klkb1 & -0.489 & $2.81 \mathrm{E}-7$ & -0.474 & $1.33 \mathrm{E}-7$ \\
\hline Slc25a39 & 0.488 & $3.03 \mathrm{E}-7$ & 0.365 & $7.57 \mathrm{E}-5$ \\
\hline Jun & 0.483 & $4.07 \mathrm{E}-7$ & 0.513 & $7.05 \mathrm{E}-9$ \\
\hline$K l b$ & -0.482 & $4.52 \mathrm{E}-7$ & -0.111 & 0.244 \\
\hline Srxn1 & 0.481 & $4.64 \mathrm{E}-7$ & 0.496 & $2.75 \mathrm{E}-8$ \\
\hline Gdf15 & 0.479 & $5.36 \mathrm{E}-7$ & 0.497 & $2.54 \mathrm{E}-8$ \\
\hline Nudt9 & 0.478 & $5.75 \mathrm{E}-7$ & 0.538 & $9.35 \mathrm{E}-10$ \\
\hline Ivns1abp & 0.476 & $6.21 \mathrm{E}-7$ & 0.407 & $8.39 \mathrm{E}-6$ \\
\hline Fermt2 & -0.474 & $7.26 \mathrm{E}-7$ & -0.347 & $1.81 \mathrm{E}-4$ \\
\hline Rusc1 & 0.473 & $7.47 \mathrm{E}-7$ & 0.252 & $7.34 \mathrm{E}-3$ \\
\hline$W f d c 2$ & 0.473 & $7.52 \mathrm{E}-7$ & 0.514 & $6.72 \mathrm{E}-9$ \\
\hline Rcan1 & 0.470 & $9.09 \mathrm{E}-7$ & 0.479 & $9.12 \mathrm{E}-8$ \\
\hline Chpt1 & 0.468 & $1.01 \mathrm{E}-6$ & 0.505 & $1.33 \mathrm{E}-8$ \\
\hline Plekha1 & 0.468 & $1.03 \mathrm{E}-6$ & 0.534 & 1.32E-9 \\
\hline
\end{tabular}

Correlations were calculated with biweight midcorrelations.

200,000 SNPs spread across the genome. We used a genome-wide significant threshold of $P=3.46 \times 10^{-6}$, as determined previously for the HMDP (23). We identified a genome-wide significant locus on chromosome 11 for female mice (Fig. 4A). Although the loci did not completely overlap, we did observe a suggestive peak at chromosome 11 for hepatic TG in males (rs13481015, 48,252,297 mb, $P=1.72 \times 10^{-5}$ ) about $3 \mathrm{mb}$ from the female peak SNP $\left(\mathrm{rs} 29391827,50,962,622 \mathrm{mb}, P=7.36 \times 10^{-8}\right)$. As shown previously (24), we identified a locus for hepatic steatosis in male mice on chromosome 7 (Fig. 4B), which we did not observe for females (Fig. 4A), suggesting a difference in genetic regulation between the sexes. We have previously shown that glycerophosphodiester phosphodiesterase 1 (Gde1) is the causal gene under the chromosome 7 locus for hepatic steatosis in males (24).

TABLE 3. Correlation between human GWAS candidate genes in mouse liver and adipose with hepatic TG level

\begin{tabular}{|c|c|c|c|c|}
\hline \multirow[b]{2}{*}{ Gene } & \multicolumn{2}{|c|}{ Liver } & \multicolumn{2}{|c|}{ Adipose } \\
\hline & Female $(r)$ & Male $(r)$ & Female $(r)$ & Male $(r)$ \\
\hline$G c k r$ & -0.03 & $0.19^{a}$ & 0.13 & 0.14 \\
\hline Lyplal1 & $0.21^{a}$ & $0.27^{b}$ & -0.13 & -0.13 \\
\hline Mboat7 & $0.23^{a}$ & -0.10 & $-0.35^{b}$ & 0.03 \\
\hline Ncan & -0.14 & -0.02 & 0.13 & $0.37^{b}$ \\
\hline Pnpla3 & 0.06 & 0.07 & -0.18 & -0.05 \\
\hline Tm6sf 2 & -0.02 & 0.15 & -0.09 & $-0.25^{b}$ \\
\hline Trib1 & 0.18 & 0.04 & $0.28^{b}$ & $0.23^{a}$ \\
\hline
\end{tabular}



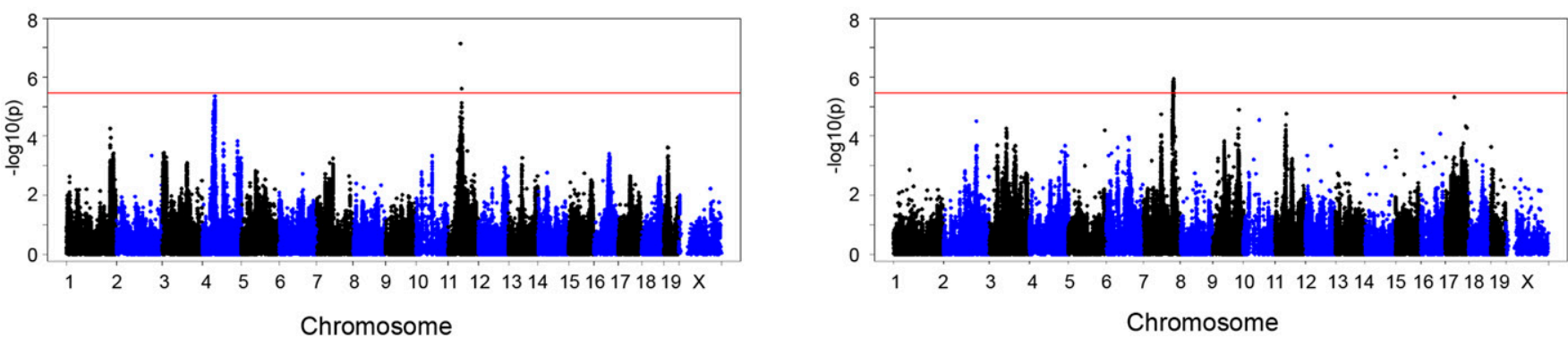

Fig. 4. Differences in major loci contributing to genetic control of hepatic TG between the sexes. A, B: Manhattan plot showing the significance (-log 10 of p) of all SNPs and hepatic TG after 8 weeks of HF/HS feeding in female (A) and male (B) mice. Genome-wide significance cut-off $\left(3.46 \times 10^{-6}\right)$ is shown by the red line.

The peak SNP for the chromosome 11 locus in females falls into a linkage disequilibrium (LD) block containing 94 genes. To identify high confidence candidate genes under the LD block, we measured global gene expression with microarray in liver and adipose tissue. In total, 49 of the genes included in the LD block were transcribed and/ or present on the array. To further screen for candidate genes, we selected genes with both a local expression quantitative trait locus [cis-expression quantitative trait locus (eQTL)] and a significant correlation between mRNA expression in liver or adipose with hepatic TG level (Table 4). Three and four liver and adipose genes showed both a significant cis-eQTL and correlation, respectively. Antioxidant 1 (Atox1) (copper chaperone) showed a significant cis-eQTL and correlation with hepatic TG in both liver and adipose. Among the candidate genes, the expression of s-phase kinase protein 1a $(S k p 1 a)$ in the liver showed the strongest correlation with hepatic TG, and was number 74 of the top 1,000 ranked genes correlating with hepatic TG. We also identified a suggestive peak on chromosome 4 for female mice $\left(r s 27776950 ; 6.76 \times 10^{-6}\right)$. Among the genes under the locus containing cis-eQTL, only inversin (Invs) (ankyrinrepeat protein) also showed a correlation with hepatic TG in the same tissue (supplemental Table S2). ABCA1 has a significant cis-eQTL in liver and the expression in adipose is correlated with hepatic TG. Although it has been shown to regulate the levels of several different lipid species in mouse livers, TG is not among them $(41,42)$.

TABLE 4. Cis-eQTL and correlations of candidate genes on chromosome 11

\begin{tabular}{lccccc}
\hline & \multicolumn{2}{c}{ Liver } & & \multicolumn{2}{c}{ Adipose } \\
\cline { 2 - 3 } \cline { 6 - 6 } Gene & cis-eQTL & $r$ & & cis-eQTL & $r$ \\
\hline Zfp354a & $9.01 \mathrm{E}-18$ & -0.20 & & $3.43 \mathrm{E}-11$ & $-0.31^{b}$ \\
Phykpl & $1.65 \mathrm{E}-37$ & 0.05 & & $4.62 \mathrm{E}-16$ & $-0.21^{a}$ \\
Hnrmpab & $6.47 \mathrm{E}-17$ & $0.20^{a}$ & & $8.63 \mathrm{E}-12$ & 0.11 \\
Skp1a & $1.23 \mathrm{E}-7$ & $0.41^{c}$ & & No & $-0.28^{b}$ \\
9530068E07Rik & $1.21 \mathrm{E}-14$ & -0.13 & & $9.08 \mathrm{E}-7$ & $0.33^{c}$ \\
Atox1 & $1.11 \mathrm{E}-30$ & $0.22^{a}$ & & $8.15 \mathrm{E}-5$ & $0.34^{c}$ \\
\hline
\end{tabular}

Correlations were calculated with biweight midcorrelations. Threshold for significant cis-eQTL: $P<1.0 \mathrm{E}-4$.

${ }^{a} P<0.05$

${ }^{b} P<0.01$.

${ }^{c} P<0.001$

\section{Gonadectomized male mice have increased hepatic TG} to $\mathrm{BF} \%$ ratio

To understand whether sex hormones play a role in the accumulation of TG in the livers of three unique strains of mice $(\mathrm{C} 3 \mathrm{H} / \mathrm{HeJ}, \mathrm{C} 57 \mathrm{BL} / 6 \mathrm{~J}$, and $\mathrm{DBA} / 2 \mathrm{~J})$, we tested to determine whether gonadectomy had an effect on hepatic TG accumulation in both sexes of these strains. The gonadectomized mice were compared with both untreated and sham operated controls. In chow-fed female mice, ovariectomy increased hepatic $\mathrm{TG}$ in $\mathrm{C} 3 \mathrm{H} / \mathrm{HeJ}$ and $\mathrm{DBA} / 2 \mathrm{~J}$ strains (Fig. 5A). In $\mathrm{C} 3 \mathrm{H} / \mathrm{HeJ}$ mice fed a $\mathrm{HF} / \mathrm{HS}$ diet for 8 weeks, ovariectomy decreased hepatic TG. Gonadectomy had no significant effect on hepatic TG in the three strains of chow-fed male mice (Fig. 5B); although the gonadectomy tended to give the same strain-specific effects in males as ovariectomy in females (Fig. 5A, B). To test to determine whether the significant effects of ovariectomy on the accumulation of TG in female livers were caused by differences in adiposity, we normalized hepatic TG to $\mathrm{BF} \%$. After the normalization, the effects of ovariectomy on hepatic TG disappeared in females (Fig. 5C). There was no significant effect of gonadectomy on hepatic TG after normalization to $\mathrm{BF} \%$ in each of the male strains (Fig. 5D). However, each male strain fed a HF/HS diet showed a trend of increased hepatic TG to $\mathrm{BF} \%$ ratio following gonadectomy. When we pooled the data from all three strains, we found a significantly higher hepatic $\mathrm{TG}$ to $\mathrm{BF} \%$ ratio in the gonadectomized male mice fed a HF/HS diet as compared with controls (Fig. 5F). In females, we saw no effect of gonadectomy after pooling the data from all three strains (Fig. 5E). The fact that testosterone seems to protect against NAFLD is supported by two previous rodent studies $(20,21)$.This suggests that some of the large variation between the male mice in hepatic TG might be explained by differences in testosterone level.

\section{DISCUSSION}

Our results have led to several findings about the differences in genetic and hormonal regulation of NAFLD in mice between sexes. By using a large set of inbred strains of mice, we clearly show that the sex differences in NAFLD are strain dependent. We also show that male laboratory 

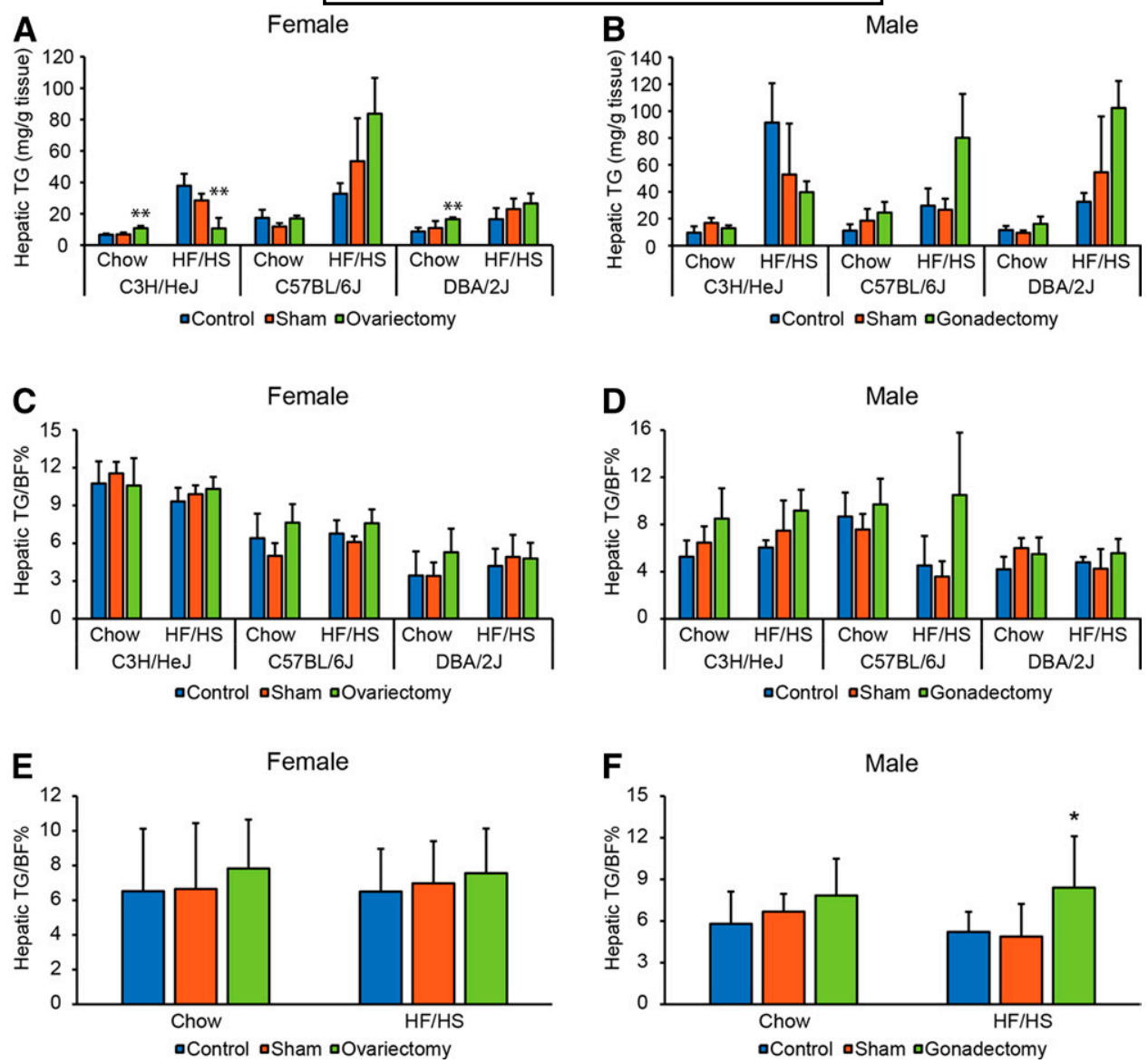

Fig. 5. Effect of gonadal hormones on hepatic TG accumulation. A, B: Hepatic TG in female (A) and male (B) strains $(\mathrm{C} 3 \mathrm{H} / \mathrm{HeJ}, \mathrm{C} 57 \mathrm{BL} / 6 \mathrm{~J}$, and DBA/2J) after ovariectomy/gonadectomy, sham operated, or untreated controls, and being maintained on chow or HF/HS diet $(n=3-4$ mice per strain, group, and sex). C, D: Hepatic TG normalized to percentage body fat (TG/BF\%) in female (C) and male (D) strains $(\mathrm{C} 3 \mathrm{H} / \mathrm{HeJ}$, C57BL/6J, and DBA/2J) after ovariectomy/gonadectomy, sham operated, or untreated controls, and being maintained on chow or HF/HS diet ( $\mathrm{n}=3-4$ mice per strain, group and sex). (E-F) Hepatic TG/BF\% in all (E) female and (F) male strains after ovariectomy/gonadectomy, sham operated or untreated controls, and being maintained on chow or HF/HS diet $(\mathrm{n}=10-12$ mice per group and sex). Results are presented as mean \pm SD. Statistical differences between groups were tested using unpaired two-tailed Student's $t$-tests. $* P<0.05$ and ** $P<0.01$ between the gonadectomized group and both controls (sham operated and untreated controls).

mice, in general, accumulate more hepatic TG than their female counterparts. Our association studies suggest that some of the differences between the sexes can be explained by accumulation of TG in specific adipose depots, and plasma HDL. Furthermore, our GWAS studies on hepatic steatosis show that male and female mice map to different loci. Finally, we show that different strains respond differently to gonadectomy in terms of NAFLD. The only observed effect that tended to be consistent between the strains of the same sex was the adverse effect of gonadectomy on hepatic steatosis after normalization to $\mathrm{BF} \%$ in male mice fed a HF/HS diet.

In general, male mice accumulate more hepatic TG than females on the HF/HS diet. The same phenomenon is observed in white men as compared with white women (4, 12). However, the genetic basis for the sex difference in hepatic steatosis has not been studied in detail. Although we generally observed more hepatic steatosis in males, in some strains, the females accumulated more hepatic TG than their male counterparts, providing strong evidence for gene-by-sex interactions. Our GWAS also showed different loci in females and males. Whereas the chromosome 11 locus for hepatic TG, which we mapped in females, might be shared between the sexes, the chromosome 7 locus, which we mapped for males, had no suggestive peak in females. The sex-specific effect on mapped hepatic steatosis loci is in accordance with what we have previously shown in a GWAS study for HOMA-IR in the same set of mice (25). Some of the explanation for different mapping between the sexes might be that some genes have a larger effect under certain circumstances.

Both human and rodent studies provide evidence for a protective effect of estrogen and testosterone on the development of fatty liver $(15-17,19-21)$. We set out to investigate the effect of gonadectomy in three different strains of both sexes. One of the main findings was that the same strains of both sexes tended to react similarly to gonadectomy with regard to hepatic TG accumulation after HF/HS feeding. Another finding was that the different strains reacted differently to the gonadectomy. Whereas C57BL/6J 
and DBA/2J tended to increase hepatic TG after gonadectomy, $\mathrm{C} 3 \mathrm{H} / \mathrm{HeJ}$ showed decreased hepatic TG levels. In females, the observed strain-specific effects of ovariectomy on hepatic TG disappeared after normalization to $\mathrm{BF} \%$. In males, on the other hand, gonadectomy showed an increased hepatic TG to $\mathrm{BF} \%$ ratio after $\mathrm{HF} / \mathrm{HS}$ diet when mice from all the strains were included in the analysis. We have previously shown in the same set of mice that ovariectomy increased insulin resistance in all the female strains on both chow and HF/HS diet (25). The fact that ovariectomy only increased hepatic TG levels in some strains, but not others, showed that there is not a one to one relationship between hepatic steatosis and insulin resistance in female mice.

Obesity, insulin resistance, and hyperlipidemia have been shown to be strongly associated to hepatic steatosis in humans of both sexes (4). As in humans, mice in the HMDP showed a strong positive correlation between hepatic steatosis and obesity, HOMA-IR, and plasma insulin levels. An interesting difference between the sexes in the HMDP is that, whereas the female mice have a fairly consistent association between hepatic steatosis and the four different fat pads, the males have a significant association with only two of the fat pads. When we used a stepwise linear regression model to describe the differences between the sexes in hepatic TG, percent gonadal fat was the phenotype that explained the largest part of the sex difference. The gonadal adipose tissue in male mice has previously been shown to exhibit a different metabolic response than subcutaneous and mesenteric adipose tissue to a HF diet (43). The authors of that study suggested that the higher levels of polyunsaturated fatty acids observed in gonadal adipose tissue are needed during spermatogenesis in the testis (43). In humans, visceral adipose tissue has been shown to be an independent risk factor for hepatic steatosis (5). We observed a stronger correlation between subcutaneous adipose tissue and hepatic TG than for the three visceral adipose tissues in our mice. The association between hyperlipidemia and hepatic steatosis is the most contradicting difference between humans and mice. Whereas humans show a positive relationship between plasma TG and hepatic TG (4), mice tend to show a negative association. Also, whereas humans show a negative correlation between HDL and hepatic steatosis, the mice show a positive trend. This difference is probably explained by the difference in lipoprotein metabolism between humans and mice. For example, in mice, but not in humans, HDL is the main cholesterol-carrying lipoprotein.

We have previously identified a locus for hepatic steatosis at chromosome 7 among male mice in the HMDP and confirmed the role of one of the genes under the locus, Gde1, in hepatic steatosis by in vivo hepatic overexpression and shRNA knockdown studies (24). Notably this locus was not observed in female mice. However, we mapped a genome-wide significant locus at chromosome 11 for hepatic TG in female mice, and identified six candidate genes based on cis-eQTLs and correlation analysis in liver and/or adipose tissue. Atoxl, which is an antioxidant and copper transporter, was the only one of the candidate genes that showed a significant cis-eQTL and correlation with hepatic TG in both liver and adipose tissue. One potential function of Atox 1 in NAFLD is its role as an antioxidant transporter, thereby sequestering of reactive oxygen species that have been implicated in mitochondrial damage (44). Skp1a is another candidate gene and is a component of SCF complexes. These ubiquitination complexes have been shown to play a role in hepatic lipid accumulation (45). Heterogenous nuclear ribonucleoprotein A/B (Hnrmpab) has been shown to play a role in apolipoprotein $\mathrm{B}$ mRNA editing in a cultured human hepatoma cell line (46). Apolipoprotein $\mathrm{B}$ is an important component of lipoproteins secreted from the liver. The candidate gene, 5-phosphohydroxyL-lysine phosphor-lyase $(P h y k p l)$, is a mitochondrial enzyme catalyzing 5-phosphonooxy-L-lysine to inorganic phosphate, 2-aminoadipate semialdehyde, and ammonia (47). Interestingly, humans with NAFLD have been shown to have a lower inorganic phosphate to total phosphate ratio, as compared with controls (48). Zinc finger protein 354a (Zfp354a) and 9530068E07Rik have no known functions related to lipids.

Human GWASs have identified several candidate genes for NAFLD (35-40) and we found that the expression of six of these in liver or adipose tissue was correlated with hepatic TG. This suggests that these genes also play a role in NAFLD in mice although not necessarily as genetic drivers. The reason that the human GWAS genes are not among our list of mapped candidate genes is likely due to the fact that different genes exhibit different genetic variation in the two organisms and/or that we do not have enough power in our HMDP to map them.

In conclusion, we have described the sex difference in hepatic TG of a large cohort of inbred strains of mice maintained under controlled environmental conditions. Our data suggests that some of the difference between the sexes in hepatic TG can be explained by differences in body fat distribution, plasma HDL, and genetic regulation, although the overall pathways appear to be conserved between sexes as judged by enrichment analysis. Our data also reveal strong gene-by-sex interactions. Ir

The authors thank Zhiqiang Zhoufor for skilled assistance with mouse experiments and Sarada Charugundla for performing biochemical assays.

\section{REFERENCES}

1. Ratziu, V., S. Bellentani, H. Cortez-Pinto, C. Day, and G. Marchesini. 2010. A position statement on NAFLD/NASH based on the EASL 2009 special conference. J. Hepatol. 53: 372-384.

2. Mahady, S. E., and J. George. 2012. Management of nonalcoholic steatohepatitis: an evidence-based approach. Clin. Liver Dis. 16: 631-645.

3. Samuel, V. T., and G. I. Shulman. 2012. Mechanisms for insulin resistance: common threads and missing links. Cell. 148: 852-871.

4. Browning, J. D., L. S. Szczepaniak, R. Dobbins, P. Nuremberg, J. D. Horton, J. C. Cohen, S. M. Grundy, and H. H. Hobbs. 2004. Prevalence of hepatic steatosis in an urban population in the United States: impact of ethnicity. Hepatology. 40: 1387-1395.

5. Park, B. J., Y. J. Kim, D. H. Kim, W. Kim, Y. J. Jung, J. H. Yoon, C. Y. Kim, Y. M. Cho, S. H. Kim, K. B. Lee, et al. 2008. Visceral adipose 
tissue area is an independent risk factor for hepatic steatosis. $J$. Gastroenterol. Hepatol. 23: 900-907.

6. Schreuder, T. C., B. J. Verwer, C. M. van Nieuwkerk, and C. J. Mulder. 2008. Nonalcoholic fatty liver disease: an overview of current insights in pathogenesis, diagnosis and treatment. World J. Gastroenterol. 14: 2474-2486.

7. Kopec, K. L., and D. Burns. 2011. Nonalcoholic fatty liver disease: a review of the spectrum of disease, diagnosis, and therapy. Nutr. Clin. Pract. 26: 565-576.

8. Anstee, Q. M., A. K. Daly, and C. P. Day. 2011. Genetic modifiers of non-alcoholic fatty liver disease progression. Biochim. Biophys. Acta. 1812: 1557-1566.

9. Day, C. P. 2002. Pathogenesis of steatohepatitis. Best Pract. Res. Clin. Gastroenterol. 16: 663-678.

10. Weston, S. R., W. Leyden, R. Murphy, N. M. Bass, B. P. Bell, M. M. Manos, and N. A. Terrault. 2005. Racial and ethnic distribution of nonalcoholic fatty liver in persons with newly diagnosed chronic liver disease. Hepatology. 41: 372-379.

11. Bambha, K., P. Belt, M. Abraham, L. A. Wilson, M. Pabst, L. Ferrell, A. Unalp-Arida, and N. Bass. 2012. Ethnicity and nonalcoholic fatty liver disease. Hepatology. 55: 769-780.

12. Graff, M., K. E. North, N. Franceschini, A. P. Reiner, M. Feitosa, J. J. Carr, P. Gordon-Larsen, M. K. Wojczynski, and I. B. Borecki. 2013. PNPLA3 gene-by-visceral adipose tissue volume interaction and the pathogenesis of fatty liver disease: the NHLBI family heart study. Int. J. Obes. 37: 432-438.

13. Loomba, R., N. Schork, C. H. Chen, R. Bettencourt, A. Bhatt, B. Ang, P. Nguyen, C. Hernandez, L. Richards, J. Salotti, et al. 2015. Heritability of hepatic fibrosis and steatosis based on a prospective twin study. Gastroenterology. 149: 1784-1793.

14. Younossi, Z. M., M. Stepanova, F. Negro, S. Hallaji, Y. Younossi, B. Lam, and M. Srishord. 2012. Nonalcoholic fatty liver disease in lean individuals in the United States. Medicine. 91: 319-327.

15. Ryu, S., B. S. Suh, Y. Chang, M. J. Kwon, K. E. Yun, H. S. Jung, C. W. Kim, B. K. Kim, Y. J. Kim, Y. Choi, et al. 2015. Menopausal stages and non-alcoholic fatty liver disease in middle-aged women. Eur. J. Obstet. Gynecol. Reprod. Biol. 190: 65-70.

16. Ogawa, Y., Y. Murata, A. Nishioka, T. Inomata, and S. Yoshida. 1998. Tamoxifen-induced fatty liver in patients with breast cancer. Lancet. 351: 725.

17. Seo, N. K., H. S. Koo, J. H. Haam, H. Y. Kim, M. J. Kim, K. C. Park, K. S. Park, and Y. S. Kim. 2015. Prediction of prevalent but not incident non-alcoholic fatty liver disease by levels of serum testosterone. $J$. Gastroenterol. Hepatol. 30: 1211-1216.

18. Shin, J. Y., S. K. Kim, M. Y. Lee, H. S. Kim, B. I. Ye, Y. G. Shin, S. K. Baik, and C. H. Chung. 2011. Serum sex hormone-binding globulin levels are independently associated with nonalcoholic fatty liver disease in people with type 2 diabetes. Diabetes Res. Clin. Pract. 94: 156-162.

19. Kim, S., H. Kwon, J. H. Park, B. Cho, D. Kim, S. W. Oh, C. M. Lee, and H. C. Choi. 2012. A low level of serum total testosterone is independently associated with nonalcoholic fatty liver disease. $B M C$ Gastroenterol. 12: 69.

20. Zhang, H., Y. Liu, L. Wang, Z. Li, H. Zhang, J. Wu, N. Rahman, Y. Guo, D. Li, N. Li, et al. 2013. Differential effects of estrogen/androgen on the prevention of nonalcoholic fatty liver disease in the male rat. J. Lipid Res. 54: 345-357.

21. Kelly, D. M., J. E. Nettleship, S. Akhtar, V. Muraleedharan, D. J. Sellers, J. C. Brooke, D. S. McLaren, K. S. Channer, and T. H. Jones. 2014. Testosterone suppresses the expression of regulatory enzymes of fatty acid synthesis and protects against hepatic steatosis in cholesterol-fed androgen deficient mice. Life Sci. 109: 95-103.

22. Parks, B. W., E. Nam, E. Org, E. Kostem, F. Norheim, S. T. Hui, C. Pan, M. Civelek, C. D. Rau, B. J. Bennett, et al. 2013. Genetic control of obesity and gut microbiota composition in response to high-fat, high-sucrose diet in mice. Cell Metab. 17: 141-152.

23. Bennett, B. J., C. R. Farber, L. Orozco, H. M. Kang, A. Ghazalpour, N. Siemers, M. Neubauer, I. Neuhaus, R. Yordanova, B. Guan, et al. 2010. A high-resolution association mapping panel for the dissection of complex traits in mice. Genome Res. 20: 281-290.

24. Hui, S. T., B. W. Parks, E. Org, F. Norheim, N. Che, C. Pan, L. W. Castellani, S. Charugundla, D. L. Dirks, N. Psychogios, et al. 2015. The genetic architecture of NAFLD among inbred strains of mice. eLife. 4: e05607.

25. Parks, B. W., T. Sallam, M. Mehrabian, N. Psychogios, S. T. Hui, F. Norheim, L. W. Castellani, C. D. Rau, C. Pan, J. Phun, et al. 2015. Genetic architecture of insulin resistance in the mouse. Cell Metab. 21: 334-346.
26. Taicher, G. Z., F. C. Tinsley, A. Reiderman, and M. L. Heiman. 2003. Quantitative magnetic resonance (QMR) method for bone and whole-body-composition analysis. Anal. Bioanal. Chem. 377: 990-1002.

27. Folch, J., M. Lees, and G. H. Sloane Stanley. 1957. A simple method for the isolation and purification of total lipides from animal tissues. J. Biol. Chem. 226: 497-509.

28. Castellani, L. W., C. N. Nguyen, S. Charugundla, M. M. Weinstein, C. X. Doan, W. S. Blaner, N. Wongsiriroj, and A. J. Lusis. 2008. Apolipoprotein AII is a regulator of very low density lipoprotein metabolism and insulin resistance. J. Biol. Chem. 283: 11633-11644.

29. Yang, H., Y. Ding, L. N. Hutchins, J. Szatkiewicz, T. A. Bell, B. J. Paigen, J. H. Graber, F. P. de Villena, and G. A. Churchill. 2009. A customized and versatile high-density genotyping array for the mouse. Nat. Methods. 6: 663-666.

30. Lippert, C., J. Listgarten, Y. Liu, C. M. Kadie, R. I. Davidson, and D. Heckerman. 2011. FaST linear mixed models for genome-wide association studies. Nat. Methods. 8: 833-835.

31. Rinella, M. E. 2015. Nonalcoholic fatty liver disease: a systematic review. JAMA. 313: 2263-2273.

32. Dennis, G., Jr., B. T. Sherman, D. A. Hosack, J. Yang, W. Gao, H. C. Lane, and R. A. Lempicki. 2003. DAVID: Database for Annotation, Visualization, and Integrated Discovery. Genome Biol. 4: P3.

33. Jay, A. G., and J. A. Hamilton. The enigmatic membrane fatty acid transporter CD36: new insights into fatty acid binding and their effects on uptake of oxidized LDL. Prostaglandins Leukot. Essent. Fatty Acids. Epub ahead of print. May 20, 2016; doi:10.1016/j. plefa.2016.05.005.

34. Katunga, L. A., P. Gudimella, J. T. Efird, S. Abernathy, T. A. Mattox, C. Beatty, T. M. Darden, K. A. Thayne, H. Alwair, A. P. Kypson, et al. 2015. Obesity in a model of gpx4 haploinsufficiency uncovers a causal role for lipid-derived aldehydes in human metabolic disease and cardiomyopathy. Mol. Metab. 4: 493-506.

35. Romeo, S., J. Kozlitina, C. Xing, A. Pertsemlidis, D. Cox, L. A. Pennacchio, E. Boerwinkle, J. C. Cohen, and H. H. Hobbs. 2008. Genetic variation in PNPLA3 confers susceptibility to nonalcoholic fatty liver disease. Nat. Genet. 40: 1461-1465.

36. Chambers, J. C., W. Zhang, J. Sehmi, X. Li, M. N. Wass, P. Van der Harst, H. Holm, S. Sanna, M. Kavousi, S. E. Baumeister, et al. 2011. Genome-wide association study identifies loci influencing concentrations of liver enzymes in plasma. Nat. Genet. 43: 1131-1138.

37. Speliotes, E. K., L. M. Yerges-Armstrong, J. Wu, R. Hernaez, L. J. Kim, C. D. Palmer, V. Gudnason, G. Eiriksdottir, M. E. Garcia, L. J. Launer, et al. 2011. Genome-wide association analysis identifies variants associated with nonalcoholic fatty liver disease that have distinct effects on metabolic traits. PLoS Genet. 7: e1001324.

38. Kozlitina, J., E. Smagris, S. Stender, B. G. Nordestgaard, H. H. Zhou, A. Tybjaerg-Hansen, T. F. Vogt, H. H. Hobbs, and J. C. Cohen. 2014. Exome-wide association study identifies a TM6SF2 variant that confers susceptibility to nonalcoholic fatty liver disease. Nat. Genet. 46: 352-356.

39. Dongiovanni, P., S. Petta, C. Maglio, A. L. Fracanzani, R. Pipitone, E. Mozzi, B. M. Motta, D. Kaminska, R. Rametta, S. Grimaudo, et al. 2015. Transmembrane 6 superfamily member 2 gene variant disentangles nonalcoholic steatohepatitis from cardiovascular disease. Hepatology. 61: 506-514.

40. Mancina, R. M., P. Dongiovanni, S. Petta, P. Pingitore, M. Meroni, R. Rametta, J. Boren, T. Montalcini, A. Pujia, O. Wiklund, et al. 2016. The MBOAT7-TMC4 variant rs641738 increases risk of nonalcoholic fatty liver disease in individuals of European descent. Gastroenterology. 150: 1219-1230.e6.

41. Bi, X., X. Zhu, M. Duong, E. Y. Boudyguina, M. D. Wilson, A. K. Gebre, and J. S. Parks. 2013. Liver ABCA1 deletion in LDLrKO mice does not impair macrophage reverse cholesterol transport or exacerbate atherogenesis. Arterioscler. Thromb. Vasc. Biol. 33: 2288-2296.

42. Chung, S., J. M. Timmins, M. Duong, C. Degirolamo, S. Rong, J. K. Sawyer, R. R. Singaraja, M. R. Hayden, N. Maeda, L. L. Rudel, et al. 2010. Targeted deletion of hepatocyte ABCA1 leads to very low density lipoprotein triglyceride overproduction and low density lipoprotein hypercatabolism. J. Biol. Chem. 285: 12197-12209.

43. Caesar, R., M. Manieri, T. Kelder, M. Boekschoten, C. Evelo, M. Muller, T. Kooistra, S. Cinti, R. Kleemann, and C. A. Drevon. 2010. A combined transcriptomics and lipidomics analysis of 
subcutaneous, epididymal and mesenteric adipose tissue reveals marked functional differences. PLoS One. 5: e11525.

44. Wei, Y., R. S. Rector, J. P. Thyfault, and J. A. Ibdah. 2008. Nonalcoholic fatty liver disease and mitochondrial dysfunction. World J. Gastroenterol. 14: 193-199.

45. Onoyama, I., A. Suzuki, A. Matsumoto, K. Tomita, H. Katagiri, Y. Oike, K. Nakayama, and K. I. Nakayama. 2011. Fbxw7 regulates lipid metabolism and cell fate decisions in the mouse liver. J. Clin. Invest. 121: 342-354.

46. Lau, P. P., H. J. Zhu, M. Nakamuta, and L. Chan. 1997. Cloning of an Apobec-1-binding protein that also interacts with apolipoprotein
B mRNA and evidence for its involvement in RNA editing. J. Biol. Chem. 272: 1452-1455.

47. Veiga-da-Cunha, M., F. Hadi, T. Balligand, V. Stroobant, and E. Van Schaftingen. 2012. Molecular identification of hydroxylysine kinase and of ammoniophospholyases acting on 5-phosphohydroxy-Llysine and phosphoethanolamine. J. Biol. Chem. 287: 7246-7255.

48. Abrigo, J. M., J. Shen, V. W. Wong, D. K. Yeung, G. L. Wong, A. M. Chim, A. W. Chan, P. C. Choi, F. K. Chan, H. L. Chan, et al. 2014. Non-alcoholic fatty liver disease: spectral patterns observed from an in vivo phosphorus magnetic resonance spectroscopy study. J. Hepatol. 60: 809-815. 